Research Article

\title{
Periodical burst error correcting codes with decoding error probability
}

\author{
Pankaj Kumar Das*, Letminthang Haokip \\ Department of Mathematical Sciences, Tezpur University, Napaam, Sonitpur, Assam-784028, India
}

(Received: 2 August 2021. Received in revised form: 22 September 2021. Accepted: 25 October 2021. Published online: 27 October 2021.)

(C) 2021 the authors. This is an open access article under the CC BY (International 4.0) license (www.creativecommons.org/licenses/by/4.0/).

\begin{abstract}
In this paper, we derive necessary and sufficient conditions for the existence of periodical burst error correcting linear codes. We also give the weight distribution of periodical burst errors and a bound on the largest attainable minimum weight by such a vector in the space of $n$-tuples. Furthermore, we provide probability of decoding error of a periodical burst error correcting code.
\end{abstract}

Keywords: syndrome; periodical burst error; weight distribution; decoding probability.

2020 Mathematics Subject Classification: 94B05, 94B65, 94B70.

\section{Introduction}

Error control codes are used to control the effect of transmission or channel errors. Identifying the channel errors and applying the corresponding error control code can avoid the correction of unwarranted errors, thus improving the efficiency of the communication system. In [12], Lange introduces a type of channel error (disturbance) called periodical burst error which is found in data transmission via power lines, data channels in close distance to electronically controlled power supply units or inverters, car electric, compact discs and CD-ROM. The periodical burst error can be defined as follows.

Definition 1.1. An s-periodical burst error of length $b$ is an n-tuple whose nonzero components are confined to distinct sets of b consecutive positions such that the sets are separated by s positions and the first component of each set is nonzero.

For example, 4-periodical burst errors of length 3 in a vector of length 16 are

\section{$1000000110000010, \quad 0101000011000001, \quad 0010100001100000$.}

The paper [12] derives conditions for detection of periodical burst errors using cyclic code. Also, two methods of error correction for this error model are examined: choosing the generator polynomial of the cyclic code by two different ways. In this paper, we consider correction of periodical burst errors using linear code and derive the necessary and sufficient conditions for existence of linear codes over $G F(q)$ that can correct all $s$-periodical burst errors of length $b$. These conditions ensure us when such a code can be constructed and when not. We denote such a code by $\boldsymbol{P}_{\boldsymbol{s}, \boldsymbol{b}} \boldsymbol{B} \boldsymbol{C}$-code. Some works in this direction for different types of errors can be found in $[1,3,5,10,11,19]$. We know that the probability of error after encoding and decoding of a code is one of the major criterion for its goodness (see Section 3.7.2 of [13]), In this context, we study the probability of decoding error $P_{D}(E)$ of an $P_{s, b} B C$-code.. We first give the weight distribution of such errors and then derive a bound on the largest attainable minimum weight by such an error in the space of all $q$-ary $n$-tuples. The result is equivalent to Plotkin bound [15] (also Lemma 4.1 of Peterson and Weldon [14]). One may refer [4, 6-8, 18] and their references for weight distribution of other types of error pattern. Applying weight distribution, we finally derive the total probability of the error pattern and decoding error probability of a binary $P_{s, b} B C$-code. Note that the weight and distance are considered in the Hamming sense only.

The organization of remaining part of the paper is as follows. Section 2 provides necessary and sufficient conditions for existence of a $q$-ary $P_{s, b} B C$-code. Examples are also given. In Section 3, we give the total probability of $s$-periodical burst errors of length $b$ in a $q$-ary $n$-tuple. This is followed by the weight distribution of the error pattern and a bound on the largest attainable minimum weight by such an error. We also give the probability of decoding error of an $P_{s, b} B C$-code in a binary symmetric channel. Section 4 is the conclusion.

\footnotetext{
*Corresponding author (pankaj4thapril@yahoo.co.in).
} 


\section{Codes correcting periodical burst errors}

In this section, we obtain necessary and sufficient conditions for the existence of a $q$-ary $P_{s, b} B C$-code. We provide examples to support the results also. To prove our results, we start with two lemmas (proof of the first one is trivial) as given below.

Lemma 2.1. Let $n, b$ and $s$ be non-negative integers such that $n \geq b+s$. If an s-periodical burst error of length $b$ starts from the $i^{\text {th }}$ position $(i=1,2, \ldots, n)$ in a vector of length $n$, let $\lambda_{i}$ denote the number of sets in which nonzero components are confined and $m_{i}$ the maximum number of nonzero positions in the error pattern. Then

$$
\lambda_{i}=\left\lceil\frac{n-i+1}{s+b}\right\rceil \text { and } m_{i}=\left\lfloor\frac{n-i+1}{s+b}\right\rfloor b+\gamma((n-i+1) \bmod (b+s)),
$$

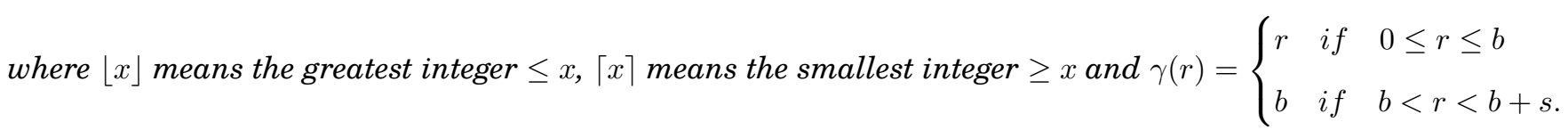

Lemma 2.2. For given non-negative integers $n, b$ and $s(n \geq b+s)$, the total number of s-periodical burst errors of length $b$ in a vector of length $n$ is $N_{s, b}=\sum_{i=1}^{n}(q-1)^{\lambda_{i}} q^{m_{i}-\lambda_{i}}$, where

$$
m_{i}=\left\lfloor\frac{n-i+1}{s+b}\right\rfloor b+\gamma((n-i+1) \quad \bmod (b+s)) \quad \text { and } \quad \lambda_{i}=\left\lceil\frac{n-i+1}{s+b}\right\rceil .
$$

Proof. An $s$-periodical burst error of length $b$ can start from the $i^{\text {th }}$ position $(i=1,2, \ldots, n)$ in a vector of length $n$. As $m_{i}$ denotes the maximum number of nonzero positions in the error pattern and $\lambda_{i}$ denotes the number of sets in which the nonzero components are confined, then by Lemma 2.1

$$
m_{i}=\left\lfloor\frac{n-i+1}{s+b}\right\rfloor b+\gamma((n-i+1) \quad \bmod (b+s)) \text { and } \lambda_{i}=\left\lceil\frac{n-i+1}{s+b}\right\rceil .
$$

Since there are $\lambda_{i}$ sets of which the first component is always nonzero, there will be $m_{i}-\lambda_{i}$ positions where the components can be any of the $q$ field elements. Therefore, the total number of $s$-periodical burst errors of length $b$ is $N_{s, b}=\sum_{i=1}^{n}(q-1)^{\lambda_{i}} q^{m_{i}-\lambda_{i}}$.

Example 2.1. Taking $n=15, b=2, s=3$ and $q=2$ in Lemma 2.2, we have $m_{1}=m_{2}=m_{3}=m_{4}=6, m_{5}=5$, $m_{6}=m_{7}=m_{8}=m_{9}=4, m_{10}=3, m_{11}=m_{12}=m_{13}=m_{14}=2, m_{15}=1$ and $\lambda_{1}=\lambda_{2}=\lambda_{3}=\lambda_{4}=\lambda_{5}=3$, $\lambda_{6}=\lambda_{7}=\lambda_{8}=\lambda_{9}=\lambda_{10}=2, \lambda_{11}=\lambda_{12}=\lambda_{13}=\lambda_{14}=\lambda_{15}=1$. Then, the total number of 3-periodical burst errors of length 2 in a vector of length 15 is $N_{3,2}=\sum_{i=1}^{15} 2^{m_{i}-\lambda_{i}}=63$. These 63 error patterns are:

$100001000010000,100001000011000,100001100010000,100001100011000,110001000010000,110001000011000$, 110001100010000, 110001100011000, 010000100001000, 010000100001100, 010000110001000, 010000110001100, 011000100001000, 011000100001100, 011000110001000, 011000110001100, 001000010000100, 001000010000110, 001000011000100, 001000011000110, 001100010000100, 001100010000110, 001100011000100, 001100011000110, 000100001000010, 000100001000011, 000100001100010, 000100001100011, 000110001000010, 000110001000011, 000110001100010, 000110001100011, 000010000100001, 000010000110001, 000011000100001, 000011000110001, 000001000010000, 000001000011000, 000001100010000, 000001100011000, 000000100001000, 000000110001000, 000000100001100, 000000110001100, 000000010000100, 0000000010000110, 000000011000100, 000000011000110, 000000001000010, 000000001100010, 000000001000011, 000000001100011, 000000000100001, 000000000110001, 000000000010000, 000000000011000, 000000000001000, 000000000001100, 000000000000100, 000000000000110, $000000000000010,000000000000011,000000000000001$.

Now, we give a necessary condition for the existence of a $q$-ary $P_{s, b} B C$-code. It is equivalent to Fire bound [9] (also Theorem 4.16 of [14]).

Theorem 2.1. For given non-negative integers $n, b$ and $s(n \geq b+s)$, a necessary condition for an $(n, k) q$-ary $P_{s, b} B C$-code is given by

$$
q^{n-k} \geq 1+N_{s, b}
$$

where $N_{s, b}$ is given in Lemma 2.2 .

Proof. As the code corrects all $s$-periodical burst errors of length $b$, all the errors must be in different cosets of the code. Thus, by Lemma 2.2, we get $q^{n-k} \geq 1+N_{s, b}$. 
Remark 2.1. From (1), we get $q^{k} \leq \frac{q^{n}}{1+N_{s, b}}$. This implies that the number of codewords of an $(n, k) q$-ary $P_{s, b} B C$-code is bounded above by $\frac{q^{n}}{1+N_{s, b}}$.

Next, we give a sufficient condition for existence of a $q$-ary $P_{s, b} B C$-code. We use the same technique that was used in Varshamov-Gilbert-Sacks Bound [17] as well as in Campopiano Bound [2] (also see Theorem 4.7 and Theorem 4.17 of [14]). This technique gives not only the sufficient condition for existence of a code, but also a method by which we can construct the code. In this technique, we construct the parity check matrix of the code by adding the columns one after another keeping in mind that the syndromes of the errors should be all nonzero and distinct.

Theorem 2.2. For given non-negative integers $n$, $b$ and $s(n \geq b+s)$, let $n=\lambda(b+s)+l$ for some non-negative integers $\lambda$ and $l$ with $0 \leq l<b+s$, a sufficient condition for an $(n, k)$-ary $P_{s, b} B C$-code is given by

$$
q^{n-k}> \begin{cases}{\left[(q-1)^{\lambda-1} q^{\lambda(b-1)}\right]\left[1+\sum_{i=1}^{n-b}(q-1)^{\lambda_{i}^{\prime}} q^{m_{i}^{\prime}-\lambda_{i}^{\prime}}\right]} & \text { if } \quad l=0 \\ {\left[(q-1)^{\lambda} q^{\lambda(b-1)+l-1}\right]\left[1+\sum_{i=1}^{n-b}(q-1)^{\lambda_{i}^{\prime}} q^{m_{i}^{\prime}-\lambda_{i}^{\prime}}\right]} & \text { if } \quad 1 \leq l \leq b \\ {\left[(q-1)^{\lambda} q^{(\lambda+1)(b-1)}\right]\left[1+\sum_{i=1}^{n-b}(q-1)^{\lambda_{i}^{\prime}} q^{m_{i}^{\prime}-\lambda_{i}^{\prime}}\right] \quad \text { if } \quad b<l<s+b,}\end{cases}
$$

where $m_{i}^{\prime}=\left\lfloor\frac{n-b-i+1}{s+b}\right\rfloor b+\gamma((n-b-i+1) \bmod (b+s))$ and $\lambda_{i}^{\prime}=\left\lceil\frac{n-b-i+1}{s+b}\right\rceil$.

Proof. The proof is shown by constructing an appropriate $(n-k) \times n$ parity-check matrix $H$ of the code. Take the first column $h_{1}$ as any nonzero ( $\left.n-k\right)$-tuple and suppose the columns $h_{2}, h_{3}, \ldots, h_{n-1}$ are added suitably to $H$. Then any (nonzero) column $h_{n}$ is added to $H$ provided that it is not a linear combination of columns of the set of immediately preceding $b-1$ columns together with the columns of previous sets of $b$ consecutive columns, along with a linear combination of the last sets of $b$ or less consecutive columns with the condition that each set is at a gap of $s$ columns. This can be written as

$$
\begin{aligned}
h_{n} \neq & \left(\sum_{i=1}^{b-1} a_{i 1} h_{n-i}+\sum_{i=0}^{b-1} b_{i 1} h_{n-(s+b)-i}+\sum_{i=0}^{b-1} b_{i 2} h_{n-2(s+b)-i}+\cdots+\sum_{i=0}^{g_{1}-1} b_{i \lambda} h_{n-\lambda(s+b)-i}\right) \\
& +\left(\sum_{i=0}^{b-1} \alpha_{i 1} h_{j^{\prime}-i}+\sum_{i=0}^{b-1} \beta_{i 1} h_{j^{\prime}-(s+b)-i}+\sum_{i=0}^{b-1} \beta_{i 2} h_{j^{\prime}-2(s+b)-i}+\cdots+\sum_{i=0}^{g_{2}-1} \beta_{i \lambda^{\prime}} h_{j^{\prime}-\lambda^{\prime}(s+b)-i}\right),
\end{aligned}
$$

where $a_{i j}, b_{i j}, \alpha_{i j}, \beta_{i j} \in G F(q) ; b_{0 i}, \alpha_{0 i}, \beta_{0 i} \neq 0 ; j^{\prime} \leq n-b ; g_{1}=\gamma(n \bmod (s+b)), g_{2}=\gamma\left(\left(n-b-j^{\prime}+1\right) \bmod (s+b)\right)$ and

$$
\lambda^{\prime}=\left\lceil\frac{n-b-j^{\prime}+1}{s+b}\right\rceil .
$$

In Expression (2), it is convenient to assume that $\sum_{i=0}^{g_{1}-1} b_{i \lambda} h_{n-\lambda(s+b)-i}=0$ and $\sum_{i=0}^{g_{2}-1} b_{i \lambda^{\prime}} h_{j^{\prime}-\lambda^{\prime}(s+b)-i}=0$ when $n$ and $n-b-j^{\prime}+1$ are multiples of $s+b$.

The condition (2) ensures that syndromes of any two s-periodical burst errors of length $b$ are distinct.

The number of linear combinations in the first bracket on right hand side (R.H.S.) of (2) is calculated as follows:

The number of ways $a_{i 1}$ 's can be chosen is $q^{b-1}$. The number of ways $b_{i j}$ 's $(j=1,2, \ldots, \lambda-1)$ can be chosen is $(q-1) q^{b-1}$. For the last summation of the first bracket, we get different combinations depending on $l$. For $l=0$, there is no term in the last summation of the first bracket. For $1 \leq l \leq b, g_{1}$ will be $l$ and $b_{i \lambda}$ 's can be chosen by $(q-1) q^{l-1}$ ways. For $b<l<s+b$, $g_{1}$ will be $b$ and $b_{i \lambda}$ 's can be chosen by $(q-1) q^{b-1}$ ways. Therefore, total number of combinations of the first bracket on R.H.S. of (2) is

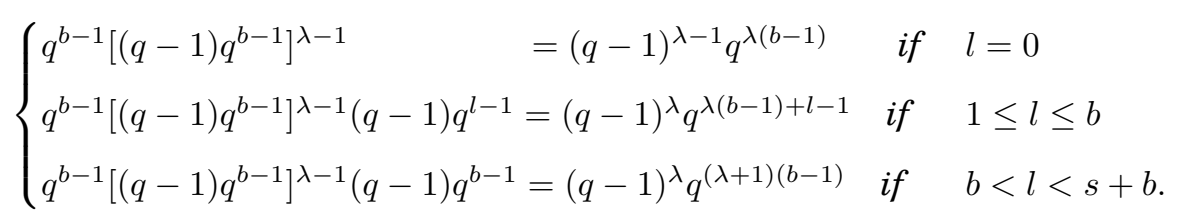

The second bracket on the R.H.S. of (2) gives the number of $s$-periodical burst errors of length $b$ in a vector of length $n-b$. This number, including the zero combination, is given by Lemma 2.2 as $1+\sum_{i=1}^{n-b}(q-1)^{\lambda_{i}^{\prime}} q^{m_{i}^{\prime}-\lambda_{i}^{\prime}}$, where

$$
m_{i}^{\prime}=\left\lfloor\frac{n-b-i+1}{s+b}\right\rfloor b+\gamma((n-b-i+1) \quad \bmod (b+s)) \text { and } \lambda_{i}^{\prime}=\left\lceil\frac{n-b-i+1}{s+b}\right\rceil .
$$


Therefore, the total number of all possible linear combinations on the R.H.S. of (2) is

$$
\left\{\begin{array}{l}
{\left[(q-1)^{\lambda-1} q^{\lambda(b-1)}\right]\left[1+\sum_{i=1}^{n-b}(q-1)^{\lambda_{i}^{\prime}} q^{m_{i}^{\prime}-\lambda_{i}^{\prime}}\right] \quad \text { if } \quad l=0} \\
{\left[(q-1)^{\lambda} q^{\lambda(b-1)+l-1}\right]\left[1+\sum_{i=1}^{n-b}(q-1)^{\lambda_{i}^{\prime}} q^{m_{i}^{\prime}-\lambda_{i}^{\prime}}\right] \quad \text { if } \quad 1 \leq l \leq b} \\
{\left[(q-1)^{\lambda} q^{(\lambda+1)(b-1)}\right]\left[1+\sum_{i=1}^{n-b}(q-1)^{\lambda_{i}^{\prime}} q^{m_{i}^{\prime}-\lambda_{i}^{\prime}}\right] \quad \text { if } \quad b<l<s+b .}
\end{array}\right.
$$

Since we can have at most $q^{n-k}-1$ nonzero columns, therefore the sufficient condition for the existence of the required code is given by

$$
q^{n-k}> \begin{cases}{\left[(q-1)^{\lambda-1} q^{\lambda(b-1)}\right]\left[1+\sum_{i=1}^{n-b}(q-1)^{\lambda_{i}^{\prime}} q^{m_{i}^{\prime}-\lambda_{i}^{\prime}}\right]} & \text { if } \quad l=0 \\ {\left[(q-1)^{\lambda} q^{\lambda(b-1)+l-1}\right]\left[1+\sum_{i=1}^{n-b}(q-1)^{\lambda_{i}^{\prime}} q^{m_{i}^{\prime}-\lambda_{i}^{\prime}}\right]} & \text { if } \quad 1 \leq l \leq b \\ {\left[(q-1)^{\lambda} q^{(\lambda+1)(b-1)}\right]\left[1+\sum_{i=1}^{n-b}(q-1)^{\lambda_{i}^{\prime}} q^{m_{i}^{\prime}-\lambda_{i}^{\prime}}\right]} & \text { if } \quad b<l<s+b .\end{cases}
$$

Remark 2.2. For $q=2$, the sufficient condition of Theorem 2.2 becomes

$$
2^{n-k}> \begin{cases}2^{\lambda(b-1)}\left[1+\sum_{i=1}^{n-b} 2^{m_{i}^{\prime}-\lambda_{i}^{\prime}}\right] & \text { if } \quad l=0 \\ 2^{\lambda(b-1)+l-1}\left[1+\sum_{i=1}^{n-b} 2^{m_{i}^{\prime}-\lambda_{i}^{\prime}}\right] & \text { if } \quad 1 \leq l \leq b \\ 2^{(\lambda+1)(b-1)}\left[1+\sum_{i=1}^{n-b} 2^{m_{i}^{\prime}-\lambda_{i}^{\prime}}\right] \quad \text { if } \quad b<l<s+b .\end{cases}
$$

Now, we give three examples to justify Theorem 2.2 corresponding to $l=0,1 \leq l \leq b$ and $b<l<s+b$.

Example 2.2. Consider $n=13, s=3, b=2$ and $q=2$ in Theorem 2.2 , then $\lambda=2, l=3$ and $\lambda_{1}^{\prime}=3, \lambda_{2}^{\prime}=\cdots=\lambda_{6}^{\prime}=2$, $\lambda_{7}^{\prime}=\cdots=\lambda_{11}^{\prime}=1, m_{1}^{\prime}=5, m_{2}^{\prime}=\cdots=m_{5}^{\prime}=4, m_{6}^{\prime}=3, m_{7}^{\prime}=\cdots=m_{10}^{\prime}=2, m_{11}^{\prime}=1$. Then

$$
\begin{aligned}
2^{n-k} & >\left[(q-1)^{\lambda} q^{(\lambda+1)(b-1)}\right]\left(1+\sum_{i=1}^{n-b}(q-1)^{\lambda_{i}^{\prime}} q^{m_{i}^{\prime}-\lambda_{i}^{\prime}}\right)=2^{(2+1)(2-1)}\left(1+\sum_{i=1}^{11} 2^{m_{i}^{\prime}-\lambda_{i}^{\prime}}\right) \\
& =8\left(1+2^{5-3}+2^{4-2} \times 4+2^{3-2}+2^{2-1} \times 4+2^{0}\right)=8 \times 32=256 .
\end{aligned}
$$

This implies that we can construct a parity check matrix

$$
H=\left[\begin{array}{lllllllllllll}
1 & 0 & 0 & 0 & 0 & 0 & 0 & 0 & 1 & 0 & 0 & 0 & 1 \\
0 & 1 & 0 & 0 & 0 & 0 & 0 & 0 & 0 & 1 & 0 & 0 & 0 \\
0 & 0 & 1 & 0 & 0 & 0 & 0 & 0 & 1 & 0 & 1 & 0 & 0 \\
0 & 0 & 0 & 1 & 0 & 0 & 0 & 0 & 0 & 0 & 0 & 1 & 0 \\
0 & 0 & 0 & 0 & 1 & 0 & 0 & 0 & 1 & 0 & 1 & 0 & 0 \\
0 & 0 & 0 & 0 & 0 & 1 & 0 & 0 & 0 & 0 & 0 & 0 & 1 \\
0 & 0 & 0 & 0 & 0 & 0 & 1 & 0 & 1 & 0 & 0 & 0 & 0 \\
0 & 0 & 0 & 0 & 0 & 0 & 0 & 1 & 0 & 0 & 1 & 0 & 0 \\
0 & 0 & 0 & 0 & 0 & 0 & 0 & 0 & 1 & 0 & 1 & 0 & 1
\end{array}\right]_{9 \times 13}
$$

of order $9 \times 13$ which gives rise to a $(13,4)$ binary linear code. It can be verified from the Error Pattern-Syndromes (Table 1) that the syndromes of all 3-periodical burst errors of length 2 are nonzero and distinct, showing that the code can correct all 3-periodical burst errors of length 2 . So, the code is a $(13,4)$ binary $P_{3,2} B C$-code. 
Table 1: Error Pattern-Syndrome.

\begin{tabular}{|ll|ll|}
\hline Error Patterns & Syndromes & Error Patterns & Syndromes \\
\hline 1000010000100 & 101011011 & 0000100001000 & 010010000 \\
1000010000110 & 101111011 & 0000100001100 & 011000011 \\
1000011000100 & 101011111 & 0000110001000 & 010011000 \\
1000011000110 & 101111111 & 0000110001100 & 011001011 \\
1100010000100 & 111011011 & 0000010000100 & 001011011 \\
1100010000110 & 111111011 & 0000010000110 & 001111011 \\
1100011000100 & 111011111 & 0000011000100 & 001011111 \\
1100011000110 & 111111111 & 0000011000110 & 001111111 \\
0100001000010 & 010100100 & 0000001000010 & 000100100 \\
0100001000011 & 110101101 & 0000001000011 & 100101101 \\
0100001100010 & 010100110 & 0000001100010 & 000100110 \\
0100001100011 & 110101111 & 0000001100011 & 100101111 \\
0110001000010 & 011100100 & 0000000100001 & 100001011 \\
0110001000011 & 111101101 & 0000000110001 & 001011110 \\
0110001100010 & 011100110 & 0000000010000 & 101010101 \\
0110001100011 & 111101111 & 0000000011000 & 111010101 \\
0010000100001 & 101001011 & 0000000001000 & 010000000 \\
0010000110001 & 000011110 & 0000000001100 & 011010011 \\
0011000100001 & 101101011 & 0000000000100 & 001010011 \\
0011000110001 & 000111110 & 0000000000110 & 001110011 \\
0001000010000 & 101110101 & 0000000000010 & 000100000 \\
0001000011000 & 111110101 & 0000000000011 & 100101001 \\
0001100010000 & 101100101 & 0000000000001 & 100001001 \\
0001100011000 & 111100101 & & \\
\hline
\end{tabular}

Example 2.3. Consider $n=11, s=3, b=2$ and $q=3$ in Theorem 2.2, then $\lambda=2, l=1$ and $\lambda_{1}^{\prime}=\cdots=\lambda_{4}^{\prime}=2$, $\lambda_{5}^{\prime}=\cdots=\lambda_{9}^{\prime}=1, m_{1}^{\prime}=m_{2}^{\prime}=m_{3}^{\prime}=4, m_{4}^{\prime}=3, m_{5}^{\prime}=\cdots=m_{8}^{\prime}=2, m_{9}^{\prime}=1$. Theorem 2.2 gives

$$
\begin{aligned}
3^{n-k} & >\left[(q-1)^{\lambda} q^{\lambda(b-1)+l-1}\right]\left(1+\sum_{i=1}^{n-b}(q-1)^{\lambda_{i}^{\prime}} q^{m_{i}^{\prime}-\lambda_{i}^{\prime}}\right)=(3-1)^{2} 3^{2(2-1)}\left(1+\sum_{i=1}^{9}(3-1)^{\lambda_{i}^{\prime}} 3^{m_{i}^{\prime}-\lambda_{i}^{\prime}}\right) \\
& =4 \times 9\left(1+4 \times 3^{4-2} \times 3+4 \times 3^{3-2}+2 \times 3^{2-1} \times 4+2 \times 3^{0}\right)=5292
\end{aligned}
$$

which implies $n-k \geq 8$. This gives rise to a ternary $(11,3)$ linear code whose parity check matrix $H$ of order $8 \times 11$ is given by

$$
H=\left[\begin{array}{lllllllllll}
1 & 0 & 0 & 0 & 0 & 0 & 0 & 0 & 2 & 0 & 0 \\
0 & 1 & 0 & 0 & 0 & 1 & 0 & 0 & 0 & 1 & 0 \\
0 & 0 & 0 & 1 & 0 & 0 & 1 & 0 & 1 & 0 & 0 \\
0 & 1 & 0 & 0 & 0 & 2 & 0 & 1 & 0 & 1 & 0 \\
0 & 0 & 0 & 2 & 0 & 0 & 1 & 0 & 0 & 0 & 1 \\
0 & 0 & 1 & 0 & 1 & 0 & 0 & 0 & 1 & 0 & 0 \\
0 & 0 & 0 & 0 & 1 & 0 & 0 & 0 & 0 & 0 & 1 \\
0 & 0 & 0 & 0 & 0 & 0 & 0 & 0 & 0 & 1 & 0
\end{array}\right]_{8 \times 11} .
$$

Here also the syndromes of all 3-periodical burst errors of length 2 are found to be nonzero and distinct, showing that the code is a ternary $(11,3) P_{3,2} B C$-code.

Example 2.4. Consider $n=10, s=3, b=2$ and $q=4$ in Theorem 2.2, then $\lambda=2, l=0$ and $\lambda_{1}^{\prime}=\cdots=\lambda_{3}^{\prime}=2, \lambda_{4}^{\prime}=\cdots=$ $\lambda_{8}^{\prime}=1, m_{1}^{\prime}=m_{2}^{\prime}=4, m_{3}^{\prime}=3, m_{4}^{\prime}=\cdots=m_{7}^{\prime}=2, m_{8}^{\prime}=1$. From Theorem 2.2, we have

$$
4^{n-k}>\left[(q-1)^{\lambda-1} q^{\lambda(b-1)}\right]\left(1+\sum_{i=1}^{n-b}(q-1)^{\lambda_{i}^{\prime}} q^{m_{i}^{\prime}-\lambda_{i}^{\prime}}\right)=(4-1) \times 4^{2(2-1)}\left[1+\sum_{i=1}^{8}(4-1)^{\lambda_{i}^{\prime}} 4^{m_{i}^{\prime}-\lambda_{i}^{\prime}}\right]=18048
$$

This implies $n-k \geq 8$. Thus we can construct a parity check matrix $H$ of order $8 \times 10$, which gives rise to a (10, 2) quaternary $P_{3,2} B C$-code: 


$$
H=\left[\begin{array}{llllllllll}
1 & 0 & 0 & 0 & 0 & 0 & 0 & 0 & 1 & 3 \\
0 & 1 & 0 & 0 & 0 & 0 & 0 & 0 & 0 & 3 \\
0 & 0 & 1 & 0 & 0 & 0 & 0 & 0 & 0 & 3 \\
0 & 0 & 0 & 1 & 0 & 0 & 0 & 0 & 0 & 0 \\
0 & 0 & 0 & 0 & 1 & 0 & 0 & 0 & 3 & 0 \\
0 & 0 & 0 & 0 & 0 & 1 & 0 & 0 & 2 & 0 \\
0 & 0 & 0 & 0 & 0 & 0 & 1 & 0 & 2 & 0 \\
0 & 0 & 0 & 0 & 0 & 0 & 0 & 1 & 0 & 1
\end{array}\right]_{8 \times 10}
$$

\section{Weight distribution and decoding error probability}

In this section, we first obtain the weight distribution of $s$-periodical burst errors of length $b$. Then, we give a bound on the largest attainable minimum weight by such an error in the space of all $q$-ary $n$-tuples. We also derive the probability of decoding error of an $P_{s, b} B C$-code in a binary symmetric channel.

Lemma 3.1. For $0 \leq j \leq n$, let $N_{s, b}(j)$ denote the number of an s-periodical burst error of length $b$ with weight $j$ in a vector of length $n$ over $G F(q)$. Then

$$
N_{s, b}(j)=\sum_{i=1}^{n}\left(\begin{array}{c}
m_{i}-\lambda_{i} \\
j-\lambda_{i}
\end{array}\right)(q-1)^{j}
$$

where $m_{i}$ and $\lambda_{i}$ are given by Lemma 2.1 .

Proof. By Lemma 2.1, $\lambda_{i}(i=1,2, \ldots, n)$ represents the number of sets where the nonzero components are confined and $m_{i}$ represents the number of nonzero components if an $s$-periodical burst error of length $b$ starts from the $i^{t h}$ position. As the first component of each set is nonzero, there will be at least $\lambda_{i}$ nonzero positions i.e., $m_{i} \geq \lambda_{i}$ and the weight of the error pattern is at least $\lambda_{i}$. Then there will be $m_{i}-\lambda_{i}$ positions where any field element can be chosen.

Therefore, to obtain an $s$-periodical burst error of length $b$ with weight $j$, we need to choose any $j-\lambda_{i}$ positions from $m_{i}-\lambda_{i}$ positions. This can be done by

$$
\left(\begin{array}{c}
m_{i}-\lambda_{i} \\
j-\lambda_{i}
\end{array}\right)
$$

ways. Note that the weight $j$ of the error pattern that starts from the $i^{t h}$ position is at least $\lambda_{i}$, so $m_{i}-\lambda_{i} \geq j-\lambda_{i} \geq 0$. Thus, the total number of $s$-periodical burst errors of length $b$ with weight $j$ is

$$
N_{s, b}(j)=\sum_{i=1}^{n}\left(\begin{array}{c}
m_{i}-\lambda_{i} \\
j-\lambda_{i}
\end{array}\right)(q-1)^{j}
$$

Remark 3.1. Observe that for given non-negative integers $n$, $b$ and $s(n \geq s+b)$, the maximum number of nonzero components in an s-periodical burst error of length b can be found when the error pattern starts from the first position. By Lemma 2.1, this number is

$$
m_{1}=\left\lfloor\frac{n}{s+b}\right\rfloor b+\gamma(n \bmod (b+s)) .
$$

So, the maximum weight of an s-periodical burst error of length $b$ can be at most $m_{1}$. Therefore, $N_{s, b}(j)=0$ for $m_{1}<j \leq n$.

Remark 3.2. We have

$$
\begin{aligned}
\sum_{j=1}^{m_{1}}\left(\begin{array}{c}
m_{i}-\lambda_{i} \\
j-\lambda_{i}
\end{array}\right)(q-1)^{j} & =\sum_{j=\lambda_{i}}^{m_{1}}\left(\begin{array}{c}
m_{i}-\lambda_{i} \\
j-\lambda_{i}
\end{array}\right)(q-1)^{j}=\sum_{\rho=0}^{m_{1}-\lambda_{i}}\left(\begin{array}{c}
m_{i}-\lambda_{i} \\
\rho
\end{array}\right)(q-1)^{\lambda_{i}+\rho} \\
& \left.=(q-1)^{\lambda_{i}} \sum_{\rho=0}^{m_{i}-\lambda_{i}}\left(\begin{array}{c}
m_{i}-\lambda_{i} \\
\rho
\end{array}\right)(q-1)^{\rho} \quad \text { because } m_{1}-\lambda_{i} \geq m_{i}-\lambda_{i}\right] \\
& =(q-1)^{\lambda_{i}} q^{m_{i}-\lambda_{i}}
\end{aligned}
$$

Therefore

$$
\sum_{i=1}^{n} \sum_{j=1}^{m_{1}}\left(\begin{array}{c}
m_{i}-\lambda_{i} \\
j-\lambda_{i}
\end{array}\right)(q-1)^{j}=\sum_{i=1}^{n}(q-1)^{\lambda_{i}} q^{m_{i}-\lambda_{i}} \quad \text { i.e., } \quad \sum_{j=1}^{m_{1}} N_{s, b}(j)=N_{s, b} .
$$


Next, we give a Plotkin's type bound for $s$-periodical burst errors of length $b$.

Theorem 3.1. The minimum weight of a vector having s-periodical burst errors of length $b$ in the space of $n$-tuples over $G F(q)$ is at most

$$
\frac{\sum_{j=1}^{m_{1}} j N_{s, b}(j)}{N_{s, b}},
$$

where $N_{s, b}$ is given by Lemma 2.2 and $N_{s, b}(j)$ by Lemma 3.1 .

Proof. By Lemma 2.2, the number of all s-periodical burst errors of length $b$ in the space of $n$-tuples over $G F(q)$ is $N_{s, b}$. From Lemma 3.1, the total weight of all $s$-periodical burst errors of length $b$ in the space of $n$-tuples over $G F(q)$ is given by $\sum_{j=1}^{m_{1}} j N_{s, b}(j)$. As the minimum weight of a vector can be at most the average weight, therefore the minimum weight of a vector having s-periodical burst errors of length $b$ in the space of $n$-tuples over $G F(q)$ is at most

$$
\frac{\sum_{j=1}^{m_{1}} j N_{s, b}(j)}{N_{s, b}} .
$$

Remark 3.3. The distance of two s-periodical burst errors of length $b$ :

$$
\begin{aligned}
& (\underbrace{x_{1}^{\prime} 0 x_{2}^{\prime} 0 x_{3}^{\prime} 0 \ldots}_{b} \underbrace{00 \ldots 0}_{s} \underbrace{x_{1}^{\prime \prime} 0 x_{2}^{\prime \prime} 0 x_{3}^{\prime \prime} 0 \ldots}_{b} \underbrace{00 \ldots 0}_{s} \ldots) \text { and } \\
& (\underbrace{0 y_{1}^{\prime} 0 y_{2}^{\prime} 0 y_{3}^{\prime} \ldots}_{b} \underbrace{00 \ldots 0}_{s} \underbrace{0 y_{1}^{\prime \prime} 0 y_{2}^{\prime \prime} 0 y_{3}^{\prime \prime} \ldots}_{b} \underbrace{00 \ldots 0}_{s} \ldots),
\end{aligned}
$$

with $x_{i}^{\prime}, y_{i}^{\prime \prime} \in G F(q) \backslash\{0\}$, gives an s-periodical burst error of length b with weight $m_{1}$. So, the minimum distance of the set with all s-periodical burst errors of length $b$ is less than or equal to $m_{1}$ and the maximum distance of the set is more than or equal to $m_{1}$.

Now, total probability of $s$-periodical burst errors of length $b$ in a binary symmetric channel is given in the following theorem.

Theorem 3.2. Let $\epsilon$ be transition probability of a memoryless binary symmetric channel. The total probability $P(E)$ of s-periodical burst errors of length $b$ in a vector of length $n$ over $G F(2)$ is given by $\sum_{i=1}^{n} \epsilon^{\lambda_{i}}(1-\epsilon)^{n-m_{i}}$, where $m_{i}$ and $\lambda_{i}$ are given in Lemma 2.1.

Proof. With the usual meaning of $m_{i}$ and $\lambda_{i}(i=1,2, \ldots, n)$ by Lemma 2.1, the number of always nonzero positions in an $s$-periodical burst error of length $b$ that starts from $i^{\text {th }}$ position is $\lambda_{i}$ and other nonzero positions can be from the remaining $m_{i}-\lambda_{i}$ positions. Therefore, the total probability of $s$-periodical burst errors of length $b$ that starts from $i^{t h}$ position is given by

$$
\sum_{j=0}^{m_{i}-\lambda_{i}}\left(\begin{array}{c}
m_{i}-\lambda_{i} \\
j
\end{array}\right) \epsilon^{\lambda_{i}+j}(1-\epsilon)^{n-\lambda_{i}-j}=\epsilon^{\lambda_{i}}(1-\epsilon)^{-\lambda_{i}}(1-\epsilon)^{n-\left(m_{i}-\lambda_{i}\right)} \sum_{j=0}^{m_{i}-\lambda_{i}}\left(\begin{array}{c}
m_{i}-\lambda_{i} \\
j
\end{array}\right) \epsilon^{j}(1-\epsilon)^{m_{i}-\lambda_{i}-j}=\epsilon^{\lambda_{i}}(1-\epsilon)^{n-m_{i}} .
$$

Varying $i$ from 1 to $n$ gives the result.

We conclude the section with the probability of decoding error for a binary $P_{s, b} B C$-code as follows.

Theorem 3.3. Let $C$ be an $(n, k)$ binary $P_{s, b} B C$-code. If $P_{D}(E)$ is the probability of decoding error of the code $C$ in a memoryless binary symmetric channel with transition probability $\epsilon$, then

$$
P_{D}(E)=1-\sum_{j=0}^{m_{1}} N_{s, b}(j) \cdot \epsilon^{j}(1-\epsilon)^{n-j},
$$

where $N_{s, b}(j)$ is given by Lemma 3.1.

Proof. An error pattern can be corrected if and only if it is a coset leader in the standard array for the code. So, the probability of correcting an error is the probability that the error is a coset leader. The probability of an $s$-periodical burst of length $b$ with weight $j$ forming one of the coset leaders is $N_{s, b}(j) \cdot \epsilon^{j}(1-\epsilon)^{n-j}$.

Therefore, the probability $P_{D}(E)$ of decoding error of the code $C$ is the probability that the error is not one of the coset leaders. So

$$
P_{D}(E)=1-\sum_{j=0}^{m_{1}} N_{s, b}(j) \cdot \epsilon^{j}(1-\epsilon)^{n-j}
$$


Remark 3.4. For $s=3, b=2$ and $\epsilon=0.01$, we determine the probability of decoding error $P_{D}(E)$ of binary $P_{s, b} B C$-codes of different lengths as follows (in Table 2).

Table 2: Table of $P_{D}(E)$.

\begin{tabular}{|c|c|c|c|}
\hline$n$ & $\lambda$ & $l$ & $P_{D}(E)$ \\
\hline 11 & 2 & 1 & 0.060 \\
12 & 2 & 2 & 0.064 \\
13 & 2 & 3 & 0.074 \\
14 & 2 & 4 & 0.085 \\
15 & 3 & 0 & 0.095 \\
16 & 3 & 1 & 0.106 \\
17 & 3 & 2 & 0.116 \\
18 & 3 & 3 & 0.127 \\
19 & 3 & 4 & 0.130 \\
20 & 4 & 0 & 0.148 \\
21 & 4 & 1 & 0.158 \\
22 & 4 & 2 & 0.169 \\
23 & 4 & 3 & 0.179 \\
\hline
\end{tabular}

We find that the probability of decoding error of an $P_{s, b} B C$-code increases as the length of the code increases. So, smaller length code is more efficient.

\section{Conclusion}

In this paper, we have studied mainly the existence and decoding error probability of periodical burst error-correcting linear codes along with the weight distribution of the error pattern. This study can be extended for low-density periodical burst errors. The concept of low-density burst was introduced by Wyner [22] by observing that disturbances normally do not exceed a certain number of digits within the $b$ consecutive positions. In addition to the common Hamming distance, there is also a burst distance introduced by Wainberg and Wolf [21]. We hope that this distance may be very useful in dealing with periodical burst errors. Linear codes with burst distance that detect/correct periodical burst errors can be investigated. Also, Singleton and Reiger bound [15,16] with respect to burst distance and a class of Maximum Distance Separable (MDS) codes were presented in [20]. Connection between these MDS codes and periodical burst errors may be interesting to explore.

\section{Acknowledgment}

The second author is supported by JRF fellowship from Council of Scientific and Industrial Research, India (File No. 09/796(0085)/2018-EMR-I).

\section{References}

[1] E. F. Assmus, H. F. Mattson, Coding and combinatorics, SIAM Rev. 16 (1974) 349-388.

[2] C. N. Campopiano, Bounds on burst error correcting codes, IRE Trans. Inform. Theory 8 (1962) 257-259.

[3] P. K. Das, Codes correcting key errors, TWMS J. Appl. Engrg. Math. 5 (2014) 110-117.

[4] P. K. Das, S. Kumar, Location and weight distribution of key errors, Mat. Vesnik 73 (2021) 43-54.

[5] B. K. Dass, P. Garg, Bounds for codes correcting/detecting repeated low-density burst errors, Discrete Math. Algorithms Appl. 4 (2012) \#1250048.

[6] C. Ding, C. Li, Infinite families of 2-designs and 3-designs from linear codes, Discrete Math. 340 (2017) 2415-2431.

[7] C. Ding, C. Li, N. Li, Z. Zhou, Three-weight cyclic codes and their weight distributions, Discrete Math. 339 (2016) $415-427$.

[8] C. Ding, J. Yang, Hamming weights in irreducible cyclic codes, Discrete Math. 313 (2013) 434-446.

[9] P. Fire, A Class of Multiple-Error-Correction Binary Codes for Non-Independent Errors, Sylvania Report RSL-E-2, Sylvania Reconnaissance Systems Laboratory, Mountain View, 1959.

[10] S. Jain, Sufficient conditions for burst error identification and correction in LRTJ-spaces, Ars Combin. 125 (2016) 129-150.

[11] S. Jain, K. P. Shum, Extended Varshamov-Gilbert-Sacks Bound for Linear Lee Weight Codes, Algebra Colloq. 19 (2012) $893-904$.

[12] N. Lange, Error Correcting Codes on Periodically Disturbed Data Channels, Proceeding of 1994 IEEE International Symposium on Information Theory, 1994, p. 33.

[13] T. K. Moon, Error Correction Coding: Mathematical Methods and Algorithms, Wiley-Interscience, New York, 2005.

[14] W. W. Peterson, E. J. Weldon, Error Correcting Codes, 2nd Edition, MIT Press, Cambridge, 1972.

[15] M. Plotkin, Binary code with specified minimum distance, IRE Trans. Inform. Theory 6 (1960) 445-450.

[16] S. Reiger, Codes for correction of clustered errors, IRE Trans. Inform. Theory 6 (1960) 16-21.

[17] G. E. Sacks, Multiple error correction by means of parity-checks, IRE Trans. Inform. Theory 4 (1958) 145-147.

[18] B. D. Sharma, B. Rohtagi, Some results on weights of vectors having $m$-repeated bursts, Cybernet. Inform. Technol. 11 (2011) 3-11.

[19] F. Temiz, V. Siap, Linear block and array codes correcting repeated CT-burst errors, Albanian J. Math. 7 (2013) 77-92.

[20] L. J. G. Villalba, A. L. S. Orozco, M. Blaum, On multiple burst-correcting MDS codes, J. Comput. Appl. Math. 295 (2016) $170-174$.

[21] S. Wainberg, J. K. Wolf, Burst decoding of binary block codes on Q-ary output channels, IEEE Trans. Inform. Theory 18 (1972) 684-686.

[22] A. D. Wyner, Low-density-burst-correcting codes, IEEE Trans. Inform. Theory 9 (1963) 124. 